\title{
A RARE CASE OF SMALL BOWEL PNEUMATOSIS INTESTINALIS PRESENTING WITH SHOCK- A CASE REPORT
}

\author{
Shrijit Kumar', Hemendra Singh², Yogesh Jhamb³, R. C. M. Kaza ${ }^{4}$, Aditya Arya ${ }^{5}$
}

${ }_{1}$ DNB Resident, Department of General Surgery, Max Super Speciality Hospital, Vaishali, Uttar Pradesh, India. ${ }^{2}$ Senior Consultant, Department of General Surgery, Max Super Speciality Hospital, Vaishali, Uttar Pradesh, India. 3 Senior Consultant, Department of General Surgery, Max Super Speciality Hospital, Vaishali, Uttar Pradesh, India. ${ }^{4}$ Academic Head and Senior Consultant, Department of General Surgery, Max Super Speciality Hospital, Vaishali, Uttar Pradesh, India. ${ }^{5}$ DNB Resident, Department of General Surgery, Max Super Speciality Hospital, Vaishali, Uttar Pradesh, India.

HOW TO CITE THIS ARTICLE: Kumar S, Singh H, Jhamb Y, et al. A rare case of small bowel pneumatosis intestinalis presenting with shock- a case report. J. Evolution Med. Dent. Sci. 2018;7(50):5400-5401, DOI: 10.14260/jemds/2018/1195

\section{PRESENTATION OF THE CASE}

An 82-year-old male presented to the emergency with complaints of abdominal distention, nausea and vomiting since the past 3 days. It was also associated with pain in the abdomen which was diffuse in nature, non-radiating with no aggravating or relieving factors. Patient gives history of decreased passage of stool and flatus since the past 2 days. Patient gives history of smoking since the past 30 years. Patient had past medical history of coronary artery disease (post-angioplasty), CKD (Chronic Kidney Disease), Hypertension and COPD (Chronic Obstructive Pulmonary Disease). Patient had a history of old Pulmonary TB for which he had undergone treatment. On admission, patient was conscious, oriented, in a state of shock with BP- 80/60 mmHg on noradrenaline support of $5 \mathrm{ml} / \mathrm{hr}$, Pulse rate- 120 beats per minute (ECG was done which showed Atrial Fibrillation with fast ventricular rate), afebrile. Chest- bilateral air entry was present with occasional ronchi, CVS- S1S2 present, P/Adistended with guarding, diffuse tenderness and absent bowel sounds. 2D Echo showed global hypokinesia with ejection fraction- 50\%. Initial resuscitation was done, and patient was optimized. Due to unstable condition of the patient and lack of improvement after initial supportive therapy patient was planned for emergency laparotomy.

\section{CLINICAL DIAGNOSIS}

1. Acute abdomen with Septic Shock.

2. Intestinal Perforation with pneumoperitoneum.

\section{DIFFERENTIAL DIAGNOSIS}

1. Perforation Peritonitis.

2. Acute Mesenteric Ischemia.

3. Acute Bowel Ischemia.

\section{PATHOLOGICAL DISCUSSION}

Initial blood investigations showed Hb- 12.2, TLC - 9690, INR1.03, Blood Urea- 30.7, Creatinine- 3.0, Serum Sodium- 136, Serum Potassium- 4.7, Blood Sugar- 107, Liver function test-

'Financial or Other Competing Interest': None.

Submission 02-11-2018, Peer Review 27-11-2018,

Acceptance 03-12-2018, Published 10-12-2018.

Corresponding Author:

Dr. Shrijit Kumar,

C/o. Mr. Ajit Kumar,

M4, $1^{\text {st }}$ Floor, Green Park Extension

Near Green Park Metro Station,

New Delhi-110016, India.

E-mail: shrijitkumar@gmail.com

DOI: $10.14260 /$ jemds $/ 2018 / 1195$ within normal limits. HbsAg was found to be reactive. X-Ray Chest was done which showed fibrocalcific opacities in mid and upper zones on both sides, calcified node in right hilum likely due to old infective aetiology, free gas under the diaphragm suggestive of pneumoperitoneum. X-Ray Abdomen showed dilated bowel loops with multiple air fluid levels.

PI was considered as either primary or secondary in origin, with a quoted $85 \%$ of cases secondary to necrotic, nonnecrotic gastrointestinal or pulmonary pathology. Although the causes of PI appear to be of multifactorial in origin, the exact causes are unknown. Two main theories have been proposed. A mechanical theory that gas dissects into the bowel wall from either the intestinal lumen or the lungs via the mediastinum due to some mechanical cause causing leading to increased pressure (Example- bowel obstruction or emphysema). 1.2,3 The second theory is the bacterial theory which proposes that gas forming bacilli enter the submucosa through the mucosal rents or increased mucosal permeability and produce gas within the mucosal wall. Small intestine is the most frequently involved part of the intestine which is generally seen secondary to other causes. Sigmoid colon is the most commonly involved part of the large intestine and is usually seen in primary type.[4] Gas filled cysts are not only confined to the gut but are also seen in omentum, mesentery, stomach, oesophagus, peritoneum, retroperitoneum and portal venous system. Rarely air-filled cysts may be seen in the lymph nodes.

In connective tissue disorders and inflammatory conditions, invasive microorganisms can breach the mucosal barrier. Over production of hydrogen, either due to bacteria or unabsorbable substrate like lactulose inside the lumen of the intestine can force it through the mucosal breaches to form the air-filled cysts. The content of these cysts has been analysed and Mujahed et al. found $89 \%$ of nitrogen in these cysts. Other gases seen in the cysts are hydrogen, oxygen and carbon dioxide. Stable patients with pneumoperitoneum should be subjected to Contrast Enhanced Computerized Tomography (CECT) scan of the abdomen.4,5,6 It is the investigation of choice which will show air filled cystic spaces within the bowel wall, retro peritoneum and portal circulation. It will also tell the status of lungs in case of chronic smokers where this condition is most commonly seen. 


\section{Bowel necrosis \\ Necrotizing enterocolitis \\ Ischemia and infarction \\ Neutropenic colitis}

2. Mucosal disruption

Peptic ulcer disease
Pyloric obstruction

Bowel obstruction

Ruptured jejunal diverticula

Surgery

Endoscopy

Biliary stent perforation

Sclerotherapy

3. Increased mucosal permeability

Immunotherapy

Graft versus host disease

Organ transplantation

Bone marrow transplantation AIDS enterocolitides

\section{Pulmonary disease \\ Asthma \\ Chronic pulmonary disease}

Sepsis

Emphysematous gastritis

Caustic ingestion

Intracatheter jejunostomy tube

Blunt abdominal trauma

Trauma of child abuse

Hirschsprung disease

Crohn disease

Ulcerative colitis

Systemic amyloidosis

Figure 1. Pathogenic Classification of Pneumatosis Intestinalis. AIDS= Acquired Immuno-Deficiency Syndrome

\section{DISCUSSION OF MANAGEMENT}

Treatment of this condition is mainly conservative. Treating the underlying condition will lead to resolution of this disease.

Medical management requires high flow oxygen, dietary modifications and sometimes antibiotics like metronidazole for a long duration. Oxygen diffuses into the cysts and forces the other gases out into the blood. Hyperbaric oxygen at 2.5 atmosphere pressure for two hours per day can reduce the duration of oxygen therapy. ${ }^{7}$

Endoscopic treatments like polypectomy should be avoided as it can lead to perforation and peritonitis.

Surgical management should be considered urgently whenever a patient presents with features of ischemic gut, intestinal obstruction, perforation, sepsis or features of peritonitis. Features of low-flow vascular states such as sepsis, congestive heart failure, use of ionotropic agents, and other causes of hypotension in the acute setting should lead to a high index of suspicion for ischemic bowel and a low threshold for surgery.

Our patient had several risk factors for the development of PI like COPD, intestinal obstruction, chronic kidney disease and history of smoking which all contributed to the development of PI. The presence of signs of peritonitis and intestinal obstruction along with sepsis associated with low vascular flow states such as sepsis, shock and the need of ionotropic support lead to immediate surgical management of this patient. 8

Intra-operatively patient was found to have pneumatosis of the small intestine and the mesentery extending from the mid jejunum to the mid ileum with one short segment of normal bowel. There was no evidence of a malrotation, bands or strictures. The small bowel mesentery had the presence of pneumatosis along its entire length from the mid jejunum to the mid ileum. The colon was entirely normal as well as the rest of the intra-peritoneal organs. There was mural thickening of the small bowel along with multiple diverticuli present on the jejunum. Small bowel resection was not done due presence of the long segment of affected bowel with areas of normal bowel in between. A pelvic drain was inserted, and the abdomen was closed after thorough bowel walk was done. Post operatively patient started tolerating diet orally and was passing stools and flatus.

\section{FINAL DIAGNOSIS}

Small Bowel Pneumatosis Intestinalis with Shock.

\section{REFERENCES}

[1] Pear BL. Pneumatosis intestinalis: a review. Radiology 1998;207(1):13-9.

[2] Home (AJR). Ajronline.org. 2018 [cited 21 October 2018].

[3] Wu LL, Yang YS, Dou Y, et al. A systematic analysis of pneumatosis cystoids intestinalis. World J Gastroenterol 2013;19(30):4973-8.

[4] Donovan S, Cernigliaro J, Dawson N. Pneumatosis intestinalis: a case report and approach to management. Article ID 571387, Case Rep Med 2011;2011: p. 5.

[5] Pneumotosis intestinalis | CT-Scan | colorectal cancer Scribd. 2018 [cited 21 October 2018].

[6] Slesser AAP, Patel PH, Das SC, et al. A rare case of segmental small bowel pneumatosis intestinalis: a case report. Int J Surg Case Rep 2011;2(7):185-7.

[7] Jenkins M, Courtney H, Pope E, et al. A case report and approach to management in pneumatosis intestinalis. Ann Med Surg (Lond) 2017;23:25-7.

[8] Sharma R, Chaudhary R, Bharti R, et al. Pneumatosis cystoides intestinalis, is the emergency surgery inevitable: case report. Translational Biomedicine 2017;8(4):136. 